\title{
Modified Boltzmann equation and extended Navier-Stokes equations
}

Cite as: Phys. Fluids 32, 022001 (2020); https://doi.org/10.1063/1.5139501

Submitted: 26 November 2019 . Accepted: 15 January 2020 . Published Online: 04 February 2020

Guofeng Han (韩国锋) (D, Xiaoli Liu (刘晓丽) (D), Jin Huang (黄进), Kumar Nawnit, and Liang Sun (孙亮)

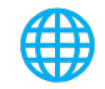

View Online

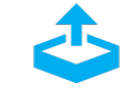

\section{ARTICLES YOU MAY BE INTERESTED IN}

A self-propelled flexible plate with a Navier slip surface

Physics of Fluids 32, 021906 (2020); https://doi.org/10.1063/1.5130698

Referee acknowledgment for 2019

Physics of Fluids 32, 020201 (2020); https://doi.org/10.1063/5.0003303

Radial deformation and disintegration of an electrified liquid jet

Physics of Fluids 32, 021701 (2020); https://doi.org/10.1063/1.5142883

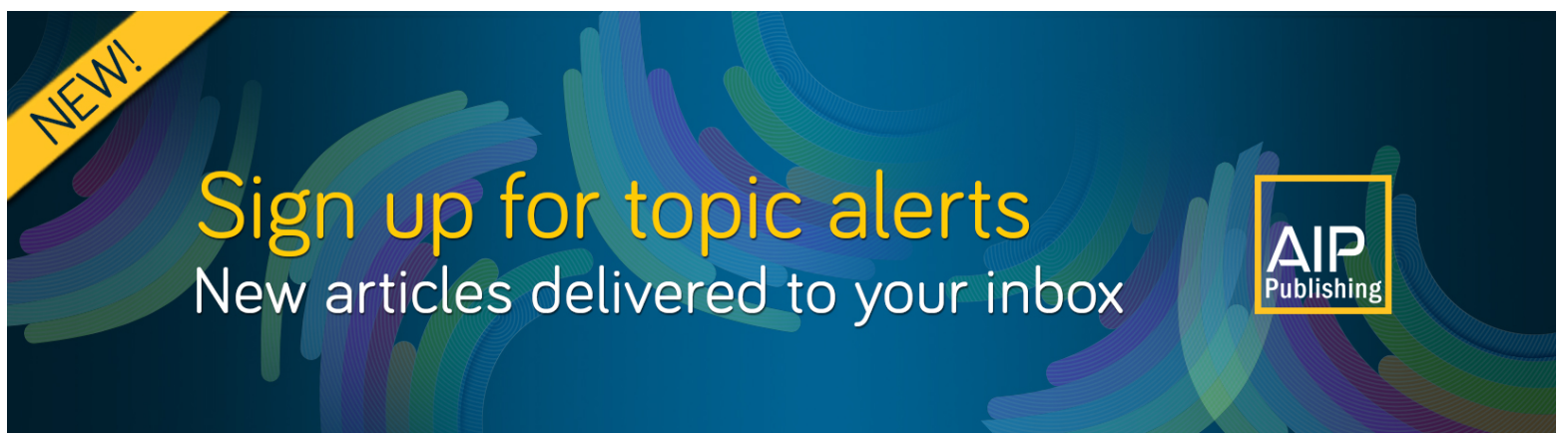




\title{
Modified Boltzmann equation and extended Navier-Stokes equations
}

\author{
Cite as: Phys. Fluids 32, 022001 (2020); doi: 10.1063/1.5139501 \\ Submitted: 26 November 2019 • Accepted: 15 January 2020 • \\ Published Online: 4 February 2020
}

\section{Guofeng Han (韩国锋), ${ }^{1}$ (D) Xiaoli Liu (刘晓丽), ${ }^{2, a)}$ (D) Jin Huang (黄进), ${ }^{2}$ Kumar Nawnit, ${ }^{2}$ and Liang Sun (孙亮)}

\author{
AFFILIATIONS \\ ${ }^{1}$ Institute of Mechanics, Chinese Academy of Sciences, Beijing 100190, China \\ ${ }^{2}$ State Key Laboratory of Hydroscience and Engineering, Tsinghua University, Beijing 100084, China \\ ${ }^{3}$ PetroChina Research Institute of Petroleum Exploration and Development, Beijing 100083, China
}

a) Author to whom correspondence should be addressed: xiaoli.liu@tsinghua.edu.cn

\begin{abstract}
We refine the derivation of the Boltzmann equation by considering that the molecules passing through the interfaces of a volume element of physical space and velocity space exhibit different velocity distribution functions and number densities. The resulting equation has a time parameter close to the relaxation time and degenerates into the conventional Boltzmann equation when this parameter takes a value of zero. By considering the macroscopic averaging of mass, momentum, and energy, the corresponding continuity, momentum, and energy equations are obtained. Compared with the extended Navier-Stokes equations, the momentum and energy equations contain additional terms to represent the external forces.
\end{abstract}

Published under license by AIP Publishing. https://doi.org/10.1063/1.5139501

\section{INTRODUCTION}

The Navier-Stokes (NS) equations govern macroscopic fluid flows. An increase in the Knudsen number (the ratio of the mean free path of the molecule to the characteristic scale) causes the NS equations to gradually fail. This situation often occurs when considering rarefied gases and microfluids. Brenner ${ }^{1}$ suggested that the definition of convection velocity in the NS equations is not suitable for such cases and proposed a bi-velocity hydrodynamic model in which the governing equations account for mass diffusion. ${ }^{2-6}$ Durst et al. ${ }^{7}$ argued that the NS equations are thermodynamically incompatible and attempted to derive new governing equations. ${ }^{8,9}$ Alternative equations have been formulated to account for the contribution of mass diffusion ignored by the macroscopic convection velocity of the NS equations, with the resulting system known as the extended NS equations. ${ }^{10}$

Many mass diffusion continuum flow models have been investigated. ${ }^{13-15}$ These models have different continuity equations, momentum equations, and energy equations compared with the NS equations. ${ }^{16-18}$ However, most equations still use the classic Newton-Fourier constitutive model. This causes the angular momentum theorem to fail for the local angular momentum. ${ }^{19}$ Therefore, alternative momentum transport constitutive models were developed. $^{20}$ It is generally believed that, when the fluid density gradient is large, the contribution of mass diffusion to motion cannot be ignored. Greenshields and Reese ${ }^{21}$ obtained better results by considering this factor when simulating shock waves. In addition, such equations have been extensively used to investigate microflows, resulting in more desirable results than existing models. ${ }^{22-2}$ Therefore, for micro-flows, the extended NS equations provide an improvement over the conventional NS equations.

However, these equations fall short of a physical basis, and phenomenological methods and the concept of the molecular mean free path are used in the derivation. For the molecular motion of gas, the Boltzmann equation is a more rigorous theory, and can, therefore, help with the accurate derivation of the continuity equation, momentum equation, and energy equation of the NS equations. To provide a more rigorous basis for the extended NS equations, Dadzie et al. ${ }^{30-32}$ modified the Boltzmann equation by adding a phenomenological term, whereas Abramov ${ }^{33}$ revised the interaction term of the Liouville equation. However, these modifications produced different results. Overall, a rigorous basis for the extended NS equations remains an open problem.

This paper describes a refinement of the conventional derivation of the Boltzmann equation, resulting in a modified Boltzmann equation. On this basis, the macroscopic mean values of conservative 
quantities during molecular collision, i.e., mass, momentum, and energy, are studied, and the continuity equation, momentum equation, and energy equation of the extended NS equations are obtained.

\section{DERIVATION OF THE MODIFIED BOLTZMANN EQUATION}

The velocity distribution function $f$ of a single-component gas molecule is a function of the spatial position vector $\mathbf{r}(x, y, z)$, the molecular velocity vector $\xi\left(\xi_{x}, \xi_{y}, \xi_{z}\right)$, and time $t$. Let $n f(\mathbf{r}, \xi, t) d \mathbf{r} d \xi$ denote the number of molecules in the velocity element $d \xi$ at $\xi$ and the volume element $d \mathbf{r}$ near the point $\mathbf{r}$ at time $t$, and let $n$ be the molecular number density, that is, the number of molecules per unit volume near point $\mathbf{r}$ at time $t$. Let $m \mathbf{F}$ be the force acting on each molecule, where $m$ is the mass of the molecule. For simplicity, we assume that $\mathbf{F}$ is independent of $\xi$. After some time interval $d t$, the number of molecules in the element $d \mathbf{r} d \xi$ increases by $\frac{\partial(n f)}{\partial t} d t d \mathbf{r} d \xi$ for two reasons. The first is the movement of molecules. The molecular velocity changes the molecule's position, and the external force changes the velocity of the molecule. The second reason is that the collision of molecules changes the velocity of the molecules. The change in the number of molecules due to collisions is $\left[\frac{\partial(n f)}{\partial t}\right]_{\text {coll }} d t d \mathbf{r} d \boldsymbol{\xi}$.

The element $d \mathbf{r} d \xi$ has six pairs of boundaries $(x, x+d x),(y$, $y+d y),(z, \mathrm{z}+d z),\left(\xi_{x}, \xi_{x}+d \xi_{x}\right),\left(\xi_{y}, \xi_{y}+d \xi_{y}\right)$, and $\left(\xi_{z}, \xi_{z}+\right.$ $\left.d \xi_{z}\right)$. By calculating the number of molecules passing through these six pairs of planes during time interval $d t$, the change in the number of molecules in element $d \mathbf{r} d \boldsymbol{\xi}$ during $d t$ because of molecular motion can be obtained. First, consider the number of molecules entering element $d \mathbf{r} d \xi$ through the plane $d y d z d \xi_{x} d \xi_{y} d \xi_{z}$ during time interval $d t$ (Fig. 1). These molecules must be located in the cylinder standing on the base $d y d z d \xi_{x} d \xi_{y} d \xi_{z}$ with the axis $\xi\left(\xi_{x}, \xi_{y}, \xi_{z}\right)$, which has a slant height of $\xi_{x} d t$. The number of molecules in this cylinder is $\left(\int_{\mathbf{r}-\xi d t}^{\mathbf{r}} n f d x^{\prime}\right) d y d z d \xi_{x} d \xi_{y} d \xi_{z}$ (to distinguish it from $\mathrm{d} x$, when calculating the number of molecules flowing into the element volume from the boundary, we use $d x^{\prime}$ to represent the differential along the $x$-direction; the notations $d y^{\prime}, d z^{\prime}, d \xi_{x}^{\prime}, d \xi_{y}^{\prime}$, and $d \xi_{z}^{\prime}$ are similarly defined). This is the number of molecules entering element $d \mathbf{r} d \boldsymbol{\xi}$ through the $x$-plane during time interval $d t$. Notably, this method differs slightly from conventional derivation methods. We consider the molecular number density and velocity distribution $f$ as a function of position. When flowing through $d y d z d \xi_{x} d \xi_{y} d \xi_{z}$, these molecules may be forced out of $(\xi, \xi+d \xi)$ through collisions with other molecules. However, other molecules may be forced into $(\xi, \xi+\mathrm{d} \xi)$ through a similar collision process. In addition, the time interval $d t$ is very short and the gas is rarefied. Therefore, there is a weak change in the distribution of molecular velocity following a collision. Similarly, the number of molecules that pass out of $d \mathbf{r} d \xi$ through the $x+d x$ plane during time interval $d t$ is $\left(\int_{\mathbf{r}-\xi_{x} d t+\mathbf{e}_{x} d x}^{\mathbf{r}+\mathbf{e}_{2}} n f d x^{\prime}\right) d y d z d \xi_{x} d \xi_{y} d \xi_{z}$, where $\mathbf{e}_{x}$ represents the unit vector in the $x$ direction. The net number of molecules entering element $d \mathbf{r} d \xi$ through a pair of planes $x$ and $x+d x$ is

$$
\begin{gathered}
\left(\int_{\mathbf{r}-\xi d t}^{\mathbf{r}} n f d x^{\prime}\right) d y d z d \xi_{x} d \xi_{y} d \xi_{z}-\left(\int_{\mathbf{r}-\xi d t+\mathbf{e}_{x} d x}^{\mathbf{r}+\mathbf{e}_{x} d x} n f d x^{\prime}\right) d y d z d \xi_{x} d \xi_{y} d \xi_{z} \\
=-\frac{\partial}{\partial x}\left(\int_{\mathbf{r}-\xi d t}^{\mathbf{r}} n f d x^{\prime}\right) d x d y d z d \xi_{x} d \xi_{y} d \xi_{z} .
\end{gathered}
$$

According to a similar method, the net number of molecules entering $d \mathbf{r} d \boldsymbol{\xi}$ through a pair of planes $\xi_{x}$ and $\xi_{x}+d \xi_{x}$ in time interval $d t$ is

$$
-\frac{\partial}{\partial \xi_{x}}\left(\int_{\xi-\mathbf{F} d t}^{\xi} n f d \xi^{\prime}\right) d x d y d z d \xi_{x} d \xi_{y} d \xi_{z} .
$$

Therefore, the net number of molecules entering $d \mathbf{r} d \boldsymbol{\xi}$ through the six pairs of planes in time interval $d t$ is

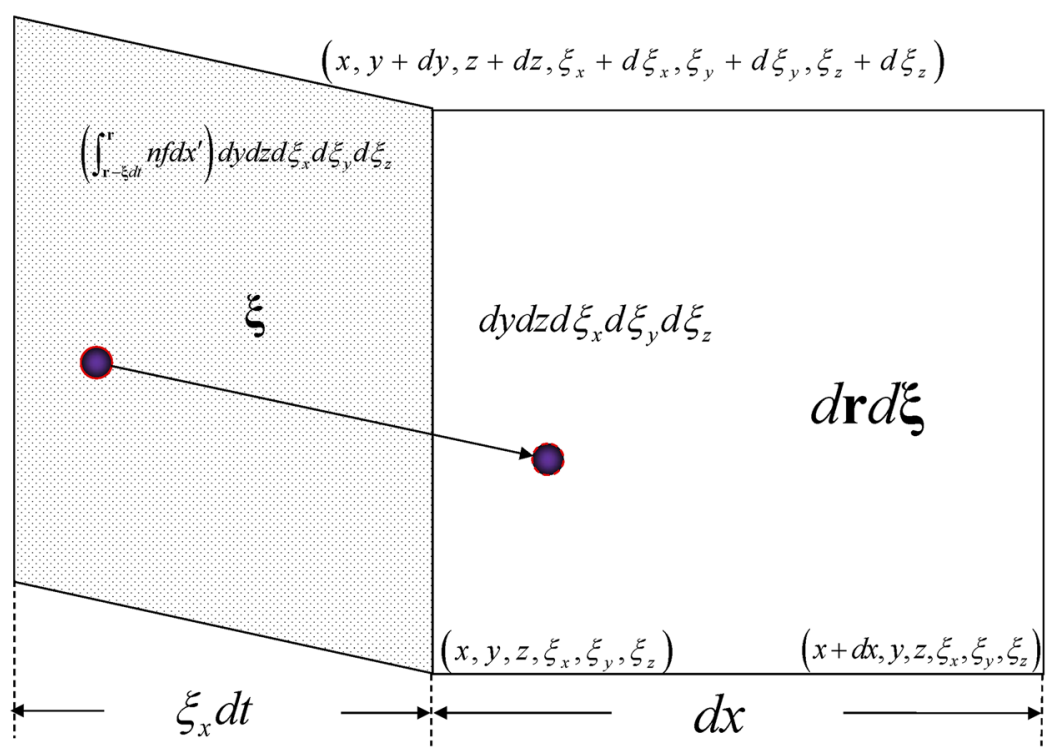

FIG. 1. Explanatory figure for the molecular motion into an element volume of physical space and velocity space (as six-dimensional space cannot be represented graphically, the area element $d y d z d \xi_{x} d \xi_{y} d \xi_{z}$ is represented by a line segment). 


$$
-\left[\begin{array}{l}
\frac{\partial}{\partial x}\left(\int_{\mathbf{r}-\xi d t}^{\mathbf{r}} n f d x^{\prime}\right)+\frac{\partial}{\partial y}\left(\int_{\mathbf{r}-\xi d t}^{\mathbf{r}} n f d y^{\prime}\right)+\frac{\partial}{\partial z}\left(\int_{\mathbf{r}-\xi d t}^{\mathbf{r}} n f d z^{\prime}\right) \\
+\frac{\partial}{\partial \xi_{x}}\left(\int_{\xi-\mathbf{F} d t}^{\xi} n f d \xi_{x}^{\prime}\right)+\frac{\partial}{\partial \xi_{y}}\left(\int_{\xi-\mathbf{F} d t}^{\xi} n f d \xi_{y}^{\prime}\right)+\frac{\partial}{\partial \xi_{z}}\left(\int_{\xi-\mathbf{F} d t}^{\xi} n f d \xi_{z}^{\prime}\right)
\end{array}\right] d x d y d z d \xi_{x} d \xi_{y} d \xi_{z} .
$$

Because the macroscopic quantities are all based on the average of a volume that is sufficiently large in microscopic terms but sufficiently small in macroscopic terms, it takes time to reach the local equilibrium. This time is represented by $d t$ in Eq. (3), which should be close to the relaxation time. Considering that it cannot be equal to $0, d t$ is replaced by the symbol $\tau$. As there is no chance of confusion, the differential symbols $d x^{\prime}, d y^{\prime}, d z^{\prime}, d \xi_{x}^{\prime}, d \xi_{y}^{\prime}$, and $d \xi_{z}^{\prime}$ are replaced by $d x, d y, d z, d \xi_{x}, d \xi_{y}$, and $d \xi_{z}$, respectively, in Eq. (4). Thus, we obtain the following equation:

$$
\begin{aligned}
\frac{\partial}{\partial t}(n f) & +\frac{\partial}{\tau \partial x}\left(\int_{\mathbf{r}-\xi \tau}^{\mathbf{r}} n f d x\right)+\frac{\partial}{\tau \partial y}\left(\int_{\mathbf{r}-\xi \tau}^{\mathbf{r}} n f d y\right)+\frac{\partial}{\tau \partial z}\left(\int_{\mathbf{r}-\xi \tau}^{\mathbf{r}} n f d z\right) \\
& +\frac{\partial}{\tau \partial \xi_{x}}\left(\int_{\xi-\mathbf{F} \tau}^{\xi} n f d \xi_{x}\right)+\frac{\partial}{\tau \partial \xi_{y}}\left(\int_{\xi-\mathbf{F} \tau}^{\xi} n f d \xi_{y}\right) \\
& +\frac{\partial}{\tau \partial \xi_{z}}\left(\int_{\xi-\mathbf{F} \tau}^{\xi} n f d \xi_{z}\right)=\left[\frac{\partial(n f)}{\partial t}\right]_{\text {coll }} .
\end{aligned}
$$

Equation (4) is the modified Boltzmann equation. The first term on the left-hand side represents the change in the velocity distribution function over time, and the other terms on the left-hand side represent the variation due to molecular motion and external forces. The change due to molecular collisions is represented on the right-hand side. The main difference between Eq. (4) and the conventional Boltzmann equation is that our formulation considers the variational property of the molecules passing through the boundaries of the control volume from different positions and times. There is a time parameter $\tau$ in the modified Boltzmann equation. When this parameter approaches 0 , the Boltzmann equation can be obtained. Although the exact value of this parameter is not known, it is certainly close to the relaxation time of the local equilibrium. This time should not be too short; otherwise, the local equilibrium cannot be reached. It also cannot be too long; otherwise, the properties of the surrounding molecules will have changed significantly.

The Liouville equation is a dynamic equation for the microscopic motion of molecular groups. When the reduced velocity distribution function of one particle in a dilute gas is considered, the Boltzmann equation can be obtained. The Liouville equation has different characteristic scales from the equations proposed here. The spatial characteristic scale of the Liouville equation is the force range of the molecular interaction, and the temporal characteristic scale is the duration of a collision. The spatial characteristic scale of the modified Boltzmann equation is the mean free path of the molecule. The element $d \mathbf{r} d \boldsymbol{\xi}$ must contain a sufficient number of molecules while remaining macroscopically small. The temporal characteristic scale is the relaxation time required to reach the local equilibrium. Therefore, the modified Boltzmann formulation is a mesoscopic scale equation, which is the result of averaging the motion of molecules governed by the Liouville equation on a mesoscopic scale.

\section{EQUATIONS OF CONSERVED QUANTITIES AFTER COLLISION}

Multiplying both sides of Eq. (4) by $\phi d \xi_{x} d \xi_{y} d \xi_{z}$ in which $\phi$ is a function that is only related to the molecular velocity and is independent of the molecular position $\mathbf{r}$ and time $t$, and taking the integral over $d \xi_{x} d \xi_{y} d \xi_{z}$ gives the following equation:

$$
\begin{aligned}
\frac{\partial}{\partial t} \int & n \phi f d \xi-\int\left(\int_{\xi-\mathbf{F} \tau}^{\xi} n f d \xi_{x} \frac{\partial \phi}{\tau \partial \xi_{x}}+\int_{\xi-\mathbf{F} \tau}^{\xi} n f d \xi_{y} \frac{\partial \phi}{\tau \partial \xi_{y}}\right. \\
& \left.+\int_{\xi-\mathbf{F} \tau}^{\xi} n f d \xi_{z} \frac{\partial \phi}{\tau \partial \xi_{z}}\right) d \xi+\frac{\partial}{\partial x} \int \frac{\phi}{\tau}\left(\int_{\mathbf{r}-\xi \tau}^{\mathbf{r}} n f d x\right) d \xi \\
& +\frac{\partial}{\partial y} \int \frac{\phi}{\tau}\left(\int_{\mathbf{r}-\xi \tau}^{\mathbf{r}} n f d y \mathbf{r}\right) d \xi+\frac{\partial}{\partial z} \int \frac{\phi}{\tau}\left(\int_{\mathbf{r}-\xi \tau}^{\mathbf{r}} n f d z\right) d \xi \\
= & \int \phi\left[\frac{\partial(n f)}{\partial t}\right]_{\text {coll }} d \xi .
\end{aligned}
$$

In the derivation of this expression, we use the fact that the variable $\phi$ is independent of position and time and apply integration by parts. Equation (5) controls the transport of the molecular property $\phi$. When $\phi$ is conservative during a collision, the right-hand side of Eq. (5) is 0 . Obviously, the mass, momentum, and energy satisfy this condition. Therefore, when $\phi=m$, Eq. (5) can be transformed into

$$
\begin{aligned}
\frac{\partial \rho}{\partial t}+ & \frac{\partial}{\partial x} \int \frac{1}{\tau}\left(\int_{\mathbf{r}-\boldsymbol{\xi} \tau}^{\mathbf{r}} \rho f d x\right) d \boldsymbol{\xi}+\frac{\partial}{\partial y} \int \frac{1}{\tau}\left(\int_{\mathbf{r}-\boldsymbol{\xi} \tau}^{\mathbf{r}} \rho f d y\right) d \boldsymbol{\xi} \\
& +\frac{\partial}{\partial z} \int \frac{1}{\tau}\left(\int_{\mathbf{r}-\boldsymbol{\xi} \tau}^{\mathbf{r}} \rho f d z\right) d \boldsymbol{\xi}=0,
\end{aligned}
$$

where $\rho=n m$ is the gas density. Equation (6) is the transport equation for the gas mass. When $\varphi=m \xi$, Eq. (5) becomes

$$
\begin{aligned}
\frac{\partial}{\partial t}( & \rho \mathbf{v})-\int\left(\int_{\xi-\mathbf{F} \tau}^{\xi} \rho f d \xi_{x} \frac{\mathbf{e}_{x}}{\tau}+\int_{\xi-\mathbf{F} \tau}^{\xi} \rho f d \xi_{y} \frac{\mathbf{e}_{y}}{\tau}+\int_{\xi-\mathbf{F} \tau}^{\xi} \rho f d \xi_{z} \frac{\mathbf{e}_{z}}{\tau}\right) d \boldsymbol{\xi} \\
& +\frac{\partial}{\partial x} \int \frac{\xi}{\tau}\left(\int_{\mathbf{r}-\xi \tau}^{\mathbf{r}} \rho f d x\right) d \boldsymbol{\xi}+\frac{\partial}{\partial y} \int \frac{\xi}{\tau}\left(\int_{\mathbf{r}-\xi \tau}^{\mathbf{r}} \rho f d y\right) d \boldsymbol{\xi} \\
& +\frac{\partial}{\partial z} \int \frac{\xi}{\tau}\left(\int_{\mathbf{r}-\boldsymbol{\xi} \tau}^{\mathbf{r}} \rho f d z\right) d \boldsymbol{\xi}=0,
\end{aligned}
$$

where $\mathbf{v}=\int \xi f d \xi$ is the average molecular velocity, also called the convective velocity of the fluid. Equation (7) is the transport equation of the gas momentum. Substituting $\phi=\frac{1}{2} m \xi \cdot \xi$ into Eq. (5), we obtain 


$$
\begin{aligned}
& \frac{\partial}{\partial t}(\rho u)+\frac{\partial}{\partial t}\left(\frac{1}{2} \rho \mathbf{v} \cdot \mathbf{v}\right)-\int\left(\frac{\xi_{x}}{\tau} \int_{\xi-\mathbf{F} \tau}^{\xi} \rho f d \xi_{x}+\frac{\xi_{y}}{\tau} \int_{\xi-\mathbf{F} \tau}^{\xi} \rho f d \xi_{y}\right. \\
& \left.+\frac{\xi_{z}}{\tau} \int_{\xi-\mathbf{F} \tau}^{\xi} \rho f d \xi_{z}\right) d \xi+\frac{\partial}{\partial x} \int \frac{\frac{1}{2} \xi \cdot \xi}{\tau}\left(\int_{\mathbf{r}-\xi \tau}^{\mathbf{r}} \rho f d x\right) d \xi \\
& +\frac{\partial}{\partial y} \int \frac{\frac{1}{2} \boldsymbol{\xi} \cdot \boldsymbol{\xi}}{\tau}\left(\int_{\mathbf{r}-\xi \tau}^{\mathbf{r}} \rho f d y\right) d \boldsymbol{\xi}+\frac{\partial}{\partial z} \int \frac{\frac{1}{2} \boldsymbol{\xi} \cdot \boldsymbol{\xi}}{\tau}\left(\int_{\mathbf{r}-\boldsymbol{\xi} \tau}^{\mathbf{r}} \rho f d z\right) d \boldsymbol{\xi}=0 \text {, }
\end{aligned}
$$

where $\mathbf{c}=\boldsymbol{\xi}-\mathbf{v}$ is the molecular thermal velocity and $u=\int \frac{1}{2} \mathbf{c} \cdot \mathbf{c} f d \boldsymbol{\xi}$ is the thermal energy for the unit mass. Notably, the thermal energy defined here only considers the translational kinetic energy of the molecular thermal motion. Other molecular energies can easily be added without affecting the derivation.

\section{DERIVATION OF THE EXTENDED NAVIER-STOKES EQUATIONS}

Taking the Taylor expansion of $\rho \varphi$ at $\mathbf{r}$, and ignoring terms above the first power, substitution into Eq. (6) gives the following equation:

$$
\frac{\partial \rho}{\partial t}+\frac{\partial}{\partial \mathbf{r}} \cdot(\rho \mathbf{v})+\frac{\partial}{\partial \mathbf{r}} \cdot \mathbf{j}^{D}=0,
$$

where $\mathbf{j}^{D}=-\int\left[\frac{1}{2} \nabla(\rho f) \cdot \boldsymbol{\xi} \tau\right] \boldsymbol{\xi} d \boldsymbol{\xi}$. Equation (9) is consistent with the continuity equation of the extended NS equations. In this expression, $\mathbf{j}^{\mathrm{D}}$ is caused by the heterogeneous molecular density and velocity distribution. Although this term includes the effect of the fluid convection velocity, it is usually very small relative to the molecular thermal velocity. Thus, it is mainly due to the mass diffusion caused by molecular thermal motion. When the macroscopic control volume is large, the influence of mass diffusion is small, and the NS equations are applicable. When the control volume is small, the influence of the mass diffusion term cannot be ignored.

A similar treatment of $\rho \varphi$ at $\mathbf{r}$ and $\xi$ enables us to transform Eq. (7) into

$$
\frac{\partial}{\partial t}(\rho \mathbf{v})-\rho \mathbf{F}+\frac{\partial}{\partial \mathbf{r}} \cdot \mathbf{P}+\frac{\partial}{\partial \mathbf{r}} \cdot(\rho \mathbf{v v})+\mathbf{F} \int \frac{1}{2} \nabla_{\xi}(\rho f) \cdot \mathbf{F} \tau d \boldsymbol{\xi}=0,
$$

where $\mathbf{P}=\mathbf{P}^{c}+\boldsymbol{\tau}^{T}, \mathbf{P}^{c}=\int \rho \operatorname{cc} f d \boldsymbol{\xi}$, and $\boldsymbol{\tau}^{T}=-\int \frac{1}{2} \boldsymbol{\xi} \boldsymbol{\xi} \nabla(\rho f) \cdot \boldsymbol{\xi} \tau d \boldsymbol{\xi} . \nabla \boldsymbol{\xi}$ represents the gradient with respect to the velocity $\xi$. Equation (10) corresponds to the momentum equation of the extended NS equations. If the velocity distribution function $f$ is an even function of $\mathbf{c}$, the stress $\mathbf{P}^{\mathrm{c}}$ only has components along the three coordinate axes. The term $\boldsymbol{\tau}^{T}$ is caused by the heterogeneous molecular density and velocity distribution, including momentum transport caused by heterogeneous convective velocity, commonly known as shear stress, and mass transport caused by mass diffusion, that is, the additional momentum transport of the extended NS equations over that of the conventional NS equations. The conventional method of deriving the viscosity of fluid is based on the momentum transport caused by the uneven convection velocity on both sides of the interface. This is reflected in the definition of $\tau^{\mathrm{T}}$. The momentum equation obtained here differs from that of the extended NS equations reported in the literature in that the last term of Eq. (10) is an additional term for the external force. This is because the time required to define the average property of the control volume cannot be zero, and molecules of different velocities are accelerated to the same velocity interval. Assuming that Maxwell's velocity distribution law is approximately applicable, the last term can be easily calculated as $-\frac{\rho m}{2 k T} \mathbf{F}(\mathbf{v} \cdot \mathbf{F} \tau)$, where $k$ is the Boltzmann constant and $T$ is the temperature in Kelvin. This term is generally negligible compared with $\rho \mathbf{F}$.

Similarly, applying the same method as above for Eq. (8), the following can be obtained:

$$
\begin{aligned}
\frac{\partial}{\partial t}( & \rho u)+\frac{\partial}{\partial t}\left(\frac{1}{2} \rho \mathbf{v} \cdot \mathbf{v}\right)+\frac{\partial}{\partial \mathbf{r}} \cdot \mathbf{q}^{c}+\frac{\partial}{\partial \mathbf{r}} \cdot(\rho u \mathbf{v})+\frac{\partial}{\partial \mathbf{r}} \cdot\left(\frac{1}{2} \rho \mathbf{v} \cdot \mathbf{v v}\right) \\
& +\frac{\partial}{\partial \mathbf{r}} \cdot\left(\mathbf{P}^{c} \cdot \mathbf{v}\right)-\rho \mathbf{F} \cdot \mathbf{v}+\int \frac{1}{2}[\nabla \xi(\rho f) \cdot \mathbf{F} \tau] \mathbf{F} \cdot \boldsymbol{\xi} d \boldsymbol{\xi} \\
& -\frac{\partial}{\partial \mathbf{r}} \cdot \int \frac{1}{4} \boldsymbol{\xi} \cdot \boldsymbol{\xi}[\nabla(\rho f) \cdot \boldsymbol{\xi} \tau] \boldsymbol{\xi} d \boldsymbol{\xi}=0,
\end{aligned}
$$

where $\mathbf{q}^{c}=\int \frac{1}{2} \rho \mathbf{c} \cdot \mathbf{c c} f d \boldsymbol{\xi}$ is the heat flow vector. If the velocity distribution function $f$ is even with respect to $\mathbf{c}$, then $\mathbf{q}^{\mathbf{c}}=0$. Considering that the macroscopic convection velocity $\mathbf{v}$ is much smaller than its thermal velocity $\mathbf{c}, \boldsymbol{\tau}^{T} \approx-\int \frac{1}{2} \mathbf{c} \boldsymbol{\xi} \nabla(\rho f) \cdot \boldsymbol{\xi} \tau d \boldsymbol{\xi}$. The last term of Eq. (11) can be written as

$$
\begin{gathered}
\int \frac{1}{4} \boldsymbol{\xi} \cdot \boldsymbol{\xi}[\nabla(\rho f) \cdot \boldsymbol{\xi} \tau] \boldsymbol{\xi} d \boldsymbol{\xi}=\int \frac{1}{4}(\mathbf{c} \cdot \mathbf{c}+\mathbf{v} \cdot \mathbf{v})[\nabla(\rho f) \cdot \boldsymbol{\xi} \tau] \boldsymbol{\xi} d \boldsymbol{\xi} \\
\quad+\int \frac{1}{2} \mathbf{c} \boldsymbol{\xi} \nabla(\rho f) \cdot \boldsymbol{\xi} \tau d \boldsymbol{\xi} \cdot \mathbf{v}=-\mathbf{q}^{D}-\boldsymbol{\tau}^{T}
\end{gathered}
$$

where $\mathbf{q}^{D}=-\int \frac{1}{4}(\mathbf{c} \cdot \mathbf{c}+\mathbf{v} \cdot \mathbf{v})[\nabla(\rho f) \cdot \boldsymbol{\xi} \tau] \boldsymbol{\xi} d \boldsymbol{\xi}, \mathbf{q}^{T}=\mathbf{q}^{c}+\mathbf{q}^{D}$. Here, $\mathbf{q}^{\mathrm{D}}$ includes the transport of kinetic energy from molecular thermal motion and the macroscopic average kinetic energy. The causes of this transport include the heterogeneous convection velocity and mass diffusion. By defining the energy density as $E=u+\frac{1}{2} \mathbf{v} \cdot \mathbf{v}$, Eq. (11) can be modified to give

$$
\begin{gathered}
\frac{\partial}{\partial t}(\rho E)+\frac{\partial}{\partial \mathbf{r}} \cdot \mathbf{q}^{T}+\frac{\partial}{\partial \mathbf{r}} \cdot(\rho E \mathbf{v})+\frac{\partial}{\partial \mathbf{r}} \cdot(\mathbf{P} \cdot \mathbf{v})-\rho \mathbf{F} \cdot \mathbf{v} \\
+\int \frac{1}{2}\left[\nabla_{\xi}(\rho f) \cdot \mathbf{F} \tau\right] \mathbf{F} \cdot \boldsymbol{\xi} d \boldsymbol{\xi}=0 .
\end{gathered}
$$

Equation (13) is the energy transport equation. Ignoring the extra terms of the external force, i.e., the last term, Eq. (13) is consistent with the extended NS equations. Assuming that Maxwell's velocity distribution law is approximately applicable, the last term can be easily calculated as $-\frac{\rho m}{2 k T} \mathbf{F} \cdot\left(\mathbf{P}^{c}+\rho \mathbf{v v}\right) \cdot \mathbf{F} \tau$, which is generally negligible compared with $\rho \mathbf{F} \cdot \mathbf{v}$.

Neglecting the extra terms of the external forces, the momentum and energy equations of the extended NS equations are consistent in form with those of the conventional NS equations. However, in the extended NS equations, $\mathbf{q}^{T}$ and the stress tensor $\mathbf{P}$ contain additional transport components due to mass diffusion. The fundamental reason for the difference between them is that the extended NS equations consider the difference in physical properties caused by the molecules passing through the control volume interface coming from different positions. On the molecular scale, the fluid is not continuous, and the macroscopic quantities are defined as the average values of the molecules in a volume that is macroscopically sufficiently small and microscopically sufficiently large. Thus, it takes time to reach the local equilibrium. The molecules passing through 
the interface of the control volume during this time interval may come from a certain distance away and have the physical properties of the corresponding position. The conventional method of deriving physical parameters such as viscosity and diffusion coefficients using the primary theory of molecular motion is also based on such a viewpoint. Thus, microscopic discontinuity is the essential reason for the differences between the extended NS equations and the conventional form.

\section{CONCLUSIONS}

We modified the Boltzmann equation by considering the temporal and spatial variation in the velocity distribution function and number density of molecules entering the volume element of physical space and velocity space. This equation has a time parameter close to the relaxation time. As this parameter approaches 0 , this equation degenerates into the conventional Boltzmann equation. Determining the exact value of this parameter requires further study. Based on the modified Boltzmann equation, the continuity equation, momentum equation, and energy equation were obtained by considering the macroscopic mean of mass, momentum, and energy, respectively. Compared with the extended NS equations, the momentum equation and the energy equation have additional items related to the external force. Subsequent studies are required to determine which equation is more realistic. Future work will attempt to validate the proposed equations. To form a closed system of equations, it is necessary to investigate the constitutive relations of mass diffusion, momentum, and energy transport. The proposed model could then be used to investigate micro-flows.

\section{ACKNOWLEDGMENTS}

The authors acknowledge the National Natural Science Foundation of China-Outstanding Youth Foundation (Grant No. 51522903), the National Natural Science Foundation of China (Grant Nos. 11602276 and 51479094), and the Key Laboratory for Mechanics in Fluid Solid Coupling Systems of CAS for their financial support of this research.

\section{REFERENCES}

${ }^{1}$ H. Brenner, "Kinematics of volume transport," Physica A 349, 11-59 (2005).

${ }^{2}$ H. Brenner, "Navier-Stokes revisited," Physica A 349, 60-132 (2005).

${ }^{3}$ H. Brenner, "Phoresis in fluids," Phys. Rev. E 84, 066317 (2011).

${ }^{4}$ H. Brenner, "Fluid mechanics in fluids at rest," Phys. Rev. E 86, 016307 (2012).

${ }^{5}$ H. Brenner, "Beyond Navier-Stokes," Int. J. Eng. Sci. 54, 67-98 (2012).

${ }^{6} \mathrm{H}$. Brenner, "Bivelocity hydrodynamics. Diffuse mass flux vs. diffuse volume flux," Physica A 392, 558-566 (2013).

${ }^{7}$ F. Durst, J. Gomes, and R. Sambasivam, "Thermo-fluid-dynamics: Do we solve the right kind of equations?," in 5th Symposium on Turbulence, Heat and Mass Transfer, Dubrovnik, Croatia, September 25-29, 2006 (Begell House Inc., 2006), pp. 3-18.

${ }^{8}$ S. Chakraborty and F. Durst, "Derivations of extended Navier-Stokes equations from upscaled molecular transport considerations for compressible ideal gas flows: Towards extended constitutive forms," Phys. Fluids 19, 088104 (2007).
${ }^{9}$ R. Sambasivam, "Extended Navier-Stokes equations: Derivations and applications to fluid flow problems," Ph.D. thesis, University of Erlangen, Erlangen, 2013.

${ }^{10}$ A. Jaishankar and G. H. McKinley, "An analytical solution to the extended Navier-Stokes equations using the Lambert W function,” AIChE J. 60, 1413-1423 (2014).

${ }^{11}$ R. Sambasivam, S. Chakraborty, and F. Durst, "Numerical predictions of backward-facing step flows in microchannels using extended Navier-Stokes equations," Microfluid. Nanofluid. 16, 757-772 (2014).

${ }^{12}$ N. Dongari, R. Sambasivam, and F. Durst, "Extended Navier-Stokes equations and treatments of micro-channel gas flows," J. Fluid Sci. Technol. 4, 454-467 (2009).

${ }^{13}$ P. Ván, M. Pavelka, and M. Grmela, "Extra mass flux in fluid mechanics," J. Non-Equilib. Thermodyn. 42, 133-152 (2016).

${ }^{14} \mathrm{M}$. Svärd, "A new Eulerian model for viscous and heat conducting compressible flows,” Physica A 506, 350-375 (2018).

${ }^{15}$ R. Abramov and J. Otto, "Nonequilibrium diffusive gas dynamics: Poiseuille microflow," Physica D 371, 13-27 (2018).

${ }^{16}$ H. C. Oettinger, Beyond Equilibrium Thermodynamics (Wiley and Sons, 2005).

${ }^{17} \mathrm{H}$. Brenner, "Proposal of a critical test of the Navier-Stokes-Fourier paradigm for compressible fluid continua," Phys. Rev. E 87, 013014 (2013).

${ }^{18} \mathrm{H}$. Brenner, "Conduction-only transport phenomena in compressible bivelocity fluids: Diffuse interfaces and Korteweg stresses,” Phys. Rev. E 89, 043020 (2014).

${ }^{19}$ S. K. Dadzie and J. M. Reese, "Analysis of the thermomechanical inconsistency of some extended hydrodynamic models at high Knudsen number," Phys. Rev. E 85, 041202 (2012).

${ }^{20}$ M. H. L. Reddy, S. K. Dadzie, R. Ocone, M. K. Borg, and J. M. Reese, "Recasting Navier-Stokes equations," J. Phys. Commun. 3, 105009 (2019).

${ }^{21}$ C. J. Greenshields and J. M. Reese, "The structure of shock waves as a test of Brenner's modifications to the Navier-Stokes equations," J. Fluid Mech. 580, 407-429 (2007).

${ }^{22}$ N. Dongari and A. Sharma, "Pressure-driven diffusive gas flows in microchannels: From the Knudsen to the continuum regimes," Microfluid. Nanofluid. 6, 679-692 (2009).

${ }^{23}$ S. K. Dadzie and H. Brenner, "Predicting enhanced mass flow rates in gas microchannels using nonkinetic models," Phys. Rev. E 86, 036318 (2012).

${ }^{24}$ Q. Lv, X. Liu, E. Wang, and S. Wang, "Analytical solution to predicting gaseous mass flow rates of microchannels in a wide range of Knudsen numbers," Phys. Rev. E 88, 013007 (2013).

${ }^{25}$ Q. Lv, E. Wang, X. Liu, and S. Wang, "Determining the intrinsic permeability of tight porous media based on bivelocity hydrodynetics," Microfluid. Nanofluid. 16, 841-848 (2014).

${ }^{26}$ P. L. L. Walls and B. Abedian, "Bivelocity gas dynamics of micro-channel Couette flow,” Int. J. Eng. Sci. 79, 21-29 (2014).

${ }^{27}$ S. K. Dadzie and C. Christou, "Bi-velocity gas dynamics of a micro lid-driven cavity heat transfer subject to forced convection," Int. Commun. Heat Mass Transfer 78, 175-181 (2016).

${ }^{28}$ L. Geng, G. Li, P. Zitha, S. Tian, M. Sheng, and X. Fan, "A diffusion-viscous flow model for simulating shale gas transport in nano-pores," Fuel 181, 887-894 (2016).

${ }^{29}$ C. Christou and S. K. Dadzie, "On the numerical simulation of rarefied gas flows in micro-channels,” J. Phys. Commun. 2, 035002 (2018).

${ }^{30}$ S. K. Dadzie, J. M. Reese, and C. R. McInnes, "A continuum model of gas flows with localized density variations," Physica A 387, 6079-6094 (2008).

${ }^{31}$ S. K. Dadzie and R. M. Jason, "Spatial stochasticity and non-continuum effects in gas flows," Phys. Lett. A 376, 967-972 (2012).

${ }^{32}$ S. K. Dadzie, "Second law of thermodynamics in volume diffusion hydrodynamics in multicomponent gas mixtures," Phys. Lett. A 376, 3223-3228 (2012).

${ }^{33}$ R. V. Abramov, "Diffusive Boltzmann equation, its fluid dynamics, Couette flow and Knudsen layers,” Physica A 484, 532-557 (2017). 